\title{
Las periferias sintácticas en el habla coloquial de español L1 y L2 y de sueco L1: un estudio tipológico y adquisicional
}

\author{
THE LEFT AND RIGHT PERIPHERY IN SPANISH L1, SPANISH L2 AND SWEDISH \\ L1. A TYPOLOGICAL AND LANGUAGE-ACQUISITIONAL STUDY
}

\section{Lars Fant}

Universidad de Estocolmo

Resumen

Las dos últimas décadas han visto crecer el interés en las denominadas periferias sintácticas, la de izquierda así como la de derecha. Deriva la presente contribución de un proyecto que se va realizando en Estocolmo y cuyo objetivo es comparar la actuación de suecos muy proficientes en español y francés $L 2$ con la de hablantes nativos de una y otra lengua. En esta comunicación se enfocarán los usos del español L1 y L2 así como del sueco L1. Por un lado, se aplica una perspectiva tipológica al examinar en qué aspectos difieren las pautas del español L1 de las del sueco L1. Por el otro lado, se toma en cuenta la perspectiva adquisicional al formular la pregunta de hasta qué punto llega a parecerse el comportamiento de hablantes no nativos, aunque muy proficientes, a la pauta nativa. Con respecto al primer parámetro, resulta que la lengua española muestra una tendencia considerablemente mayor que la sueca a colocar elementos a la izquierda del núcleo oracional, mientras la lengua sueca arroja una preferencia, más clara todavía, por la colocación en periferia de derecha. En relación con el segundo parámetro, salta al ojo la gran similitud entre los hablantes de español L1 y L2, dándose solo pocas aunque interesantes excepciones. Además, no se puede constatar ningún indicio claro de transferencia de la L1 sueca en los hablantes de español L2.

PALABRAS CLAVE: periferia izquierda, periferia derecha, funciones pragmáticas, español L1, español L2, sueco L1, tipología lingüística, uso avanzado de segundas lenguas.
Abstract

The last two decades have seen an increasing interest in what characterizes the left and right sentence peripheries. This paper is the partial account of a research project at Stockholm University which aims at comparing highly proficient Swedish users of L2 French and Spanish with native speakers with regard to how they structure the syntactic peripheries in natural colloquial speech. In the present context, however, only Spanish and Swedish will be accounted for. The analysis contains two aspects: on the one hand, a typological study comparing native speakers of the two languages and, on the other hand, a study addressing the question of the upper limits of L2 acquisition while comparing Spanish L1 and L2 speakers, the latter having Swedish as their L1.Results addressing the first aspect show that native speakers of Spanish put more weight on the left periphery than do native speakers of Swedish, who, in turn, put considerably more weight on the right periphery, thus showing a clear typological difference. As regards the second, acquisitional aspect, the behaviour of the high-proficient L2 speakers closely resembles that of the native speakers, with only a few albeit interesting exceptions. On the other hand, no clear instances of L1 transfer can be observed in the Spanish L2 productions.

KEY WORDS: left periphery, right periphery, pragmatic functions, Spanish L1, Spanish L2, Swedish L1, linguistics typology, high-level proficiency in second language use 


\section{INTRODUCCIÓN}

La última década ha visto crecer el interés en investigar las denominadas periferias sintácticas, a saber, los componentes que se encuentran al margen izquierdo y derecho de (un supuesto) núcleo oracional. Dado que estas periferias asumen funciones discursivas más bien que puramente gramaticales, el interés científico en el tema proviene sobre todo de lingüistas dedicados al estudio de la interfaz entre gramática y pragmática, a los marcadores discursivos y, en particular, al de los procesos de gramaticalización (Beeching y Detges, 2011; Closs-Traugott, 2012).

Se basa la presente contribución en un programa científico realizado en Estocolmo, "Alta proficiencia en el usuario de la L2", cuyo objetivo es comparar la actuación de suecos muy proficientes en diversas segundas lenguas con la de hablantes nativos de estas. Una iniciativa tomada dentro del marco del programa ha sido establecer el corpus "Multitareas Londres-París-Santiago" con materiales provenientes de hablantes nativos y no nativos de inglés, francés y español. De esta base de datos ha surgido el proyecto "Las periferias sintácticas en hablantes nativos y no nativos de español y francés y en hablantes nativos del sueco", en el cual están colaborando el presente autor con dos colegas de la Universidad de Estocolmo (Inge Bartning y Rakel Österberg). En el presente contexto se enfocarán los usos del español $L 1 / L 2$ y del sueco $L 1$, dejando de lado los materiales en francés L1/L2. La Figura 1 presenta gráficamente la genealogía del presente proyecto.

Por un lado, nos proponemos aplicar una perspectiva tipológica, o sea: (1) ¿en qué aspectos se distinguen las tendencias del español L1 de las del sueco L1. Por el otro lado nos interesa la perspectiva adquisicional, concretamente: (2) ¿hasta qué punto llega a parecerse el comportamiento de hablantes no nativos, aunque muy proficientes, a la pauta nativa? 


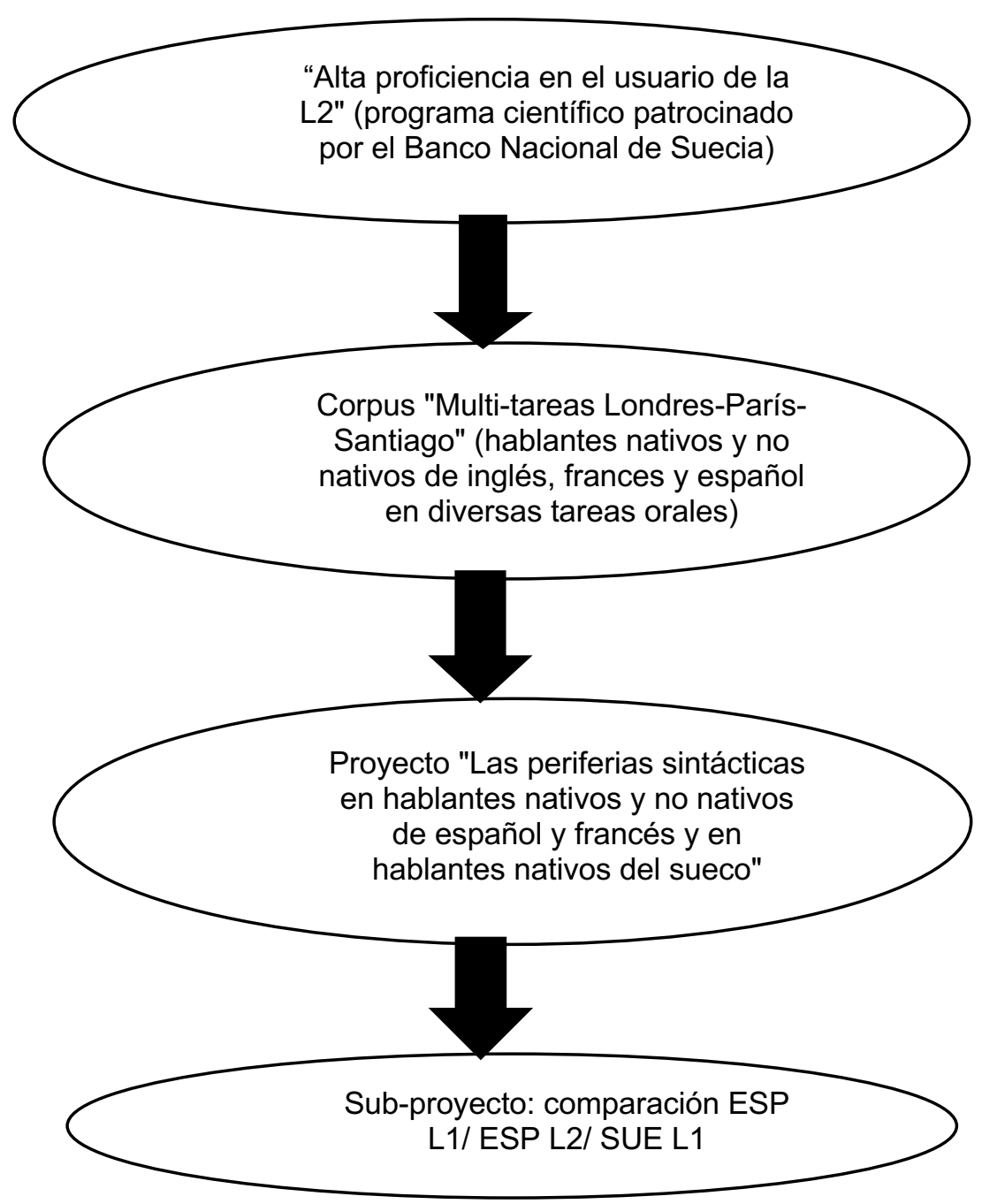

Figura 1. Desarrollo de los proyectos implicados 
Las periferias sintácticas en el habla coloquial de español L1 y L2 y de sueco L1: un estudio tipológico y adquisicional | L. Fant

Para poder contestar a estas dos preguntas de investigación hace falta, sin embargo, una labor más ardua y también más insegura, a saber, establecer una clasificación holística de los elementos que conforman la periferia izquierda (PI) así como la de derecha (PD). En la presente contribución se intentará establecer, de manera provisional, una taxonomía que sea a la vez teóricamente defendible y que permita llevar a cabo los análisis correspondientes a las preguntas de investigación.

\section{ESTADO DE LA CUESTIÓN}

La periferia izquierda oracional constituía, de hecho, el objeto de extensas investigaciones en lingüística funcionalista ya en las décadas de los 1970 y 1980, a pesar de no usarse entonces aquel mismo término. Se enfocaba ante todo la estructura informacional y sus efectos en la sintaxis, con énfasis en el fenómeno de la llamada "dislocación a la izquierda". En esa línea científica se seguía publicando estudios en las décadas siguientes, p. ej. Lambrecht (1994), Kotschi (1996) o Sedano (2013).

Los propios términos "periferia izquierda" y "periferia derecha" emergieron dentro de la lingüística generativa. Estudios representativos de esa línea son Rizzi (1997) y Cinque \& Rizzi (2008). Dentro de la lingüística hispánica se puede citar el estudio de Demonte y Fernández Soriano (2007) y, más recientemente, el de Bañeras Carrió (2016). Desde la perspectiva de la gramática generativa, la periferia izquierda se sitúa en el "sintagma infleccional" (inflectional phrase, IP), mientras el "sintagma determinante" (determiner phrase, DP) que lo gobierna no se incluye en ella.

La periferia derecha, que al principio no suscitaba el mismo interés que la de izquierda, ha recibido más atención solo en los últimos 20 años, con el creciente enfoque en la gramática construccional, la pragmática de los marcadores del discurso y los procesos de gramaticalización. El interés por las funciones discursivas ha generado trabajos sobre las periferias sintácticas tales como Morel y Danon-Boileau (1998), Closs-Traugott (2012), Detges y Waltereit (2014) y ante todo el volumen de Beeching y Detges eds. (2014). En estos estudios una postura recurrente es la atribución de una función subjetiva a la periferia izquierda y la correspondiente función intersubjetiva a la periferia derecha.

Sin embargo, a esta "hipótesis de la asimetría" (Beeching et al, 2009) le dirige ClossTraugott (2014) cierta crítica ya en el mismo volumen de Beeching y Detges (eds., 2014). A esa postura crítica se juntan también representantes del grupo Val.Es.Co (Estellés Arguedas y Pons Bordería, 2014, Salameh Jiménez, Estellés Arguedas y Pons Bordería, 2018, y Pons Bordería, en prensa) al poner en tela de juicio el propio fundamento teórico que subyace a la división en núcleo y periferias oracionales. Los mencionados autores, a cambio, abogan por la aplicación del modelo de segmentación de unidades del discurso VAM elaborado por el grupo (Briz y Grupo Val.Es.Co, 2003).

Tras este breve resumen del estado de la cuestión relativo a las periferias sintácticas podemos dirigir la mirada a nuestras dos preguntas de investigación. Con respecto a la primera de estas, se puede apreciar un cierto interés por el aspecto tipológico que se hace notar en varias contribuciones al volumen de Beeching y Detges eds. (2014). Hasta 
Las periferias sintácticas en el habla coloquial de español L1 y L2 y de sueco L1: un estudio tipológico y adquisicional | L. Fant

hoy, sin embargo, no se ha realizado ningún estudio dedicado a la comparación entre lenguas románicas y escandinavas.

En lo que se refiere al aspecto que concierne la alta competencia en segundas lenguas, se puede constatar un gran interés manifestado en los últimos años hacia los límites superiores del proceso de adquisición de una L2 y el grado de dominio que alcanza el aprendiente avanzado en la zona de interfaz entre sintaxis y discurso. Aquí se puede citar a trabajos como Hancock (2007), Hendriks y Watorek (2008), Sorace (2003), Bartning , Forsberg y Hancock (2012) así como las diversas contribuciones al volumen de Hyltenstam, Bartning y Fant eds. (2018).

\section{CONSIDERACIONES TEÓRICAS}

El presente trabajo se propone definir tipos de unidad presentes en la PI y PD respectivamente con el fin de poder aplicar la taxonomía resultante al análisis de un corpus oral bilingüe. La meta de la taxonomía es crear un sistema holístico capaz de dar cuenta de todos los tipos de elementos que aparecen en la periferia izquierda y la derecha. El procedimiento consiste en establecer categorías pragmáticas funcionales a partir de elementos gramaticalmente definidos.

Los objetivos del análisis propio, por otra parte, son los dos mencionados: (1) el tipológico comparando pautas españolas y suecas, y (2) el otro relativo al uso muy avanzado de la L2 a fin de explorar los (eventuales) límites de la adquisición.

Aunque estamos, en principio, de acuerdo con la visión de los representantes del Grupo Val.Es.Co de que la segmentación de las unidades discursivas no es compatible con una división unidimensional en núcleo, periferia izquierda y periferia derecha, hemos procedido desde otra perspectiva, al considerar las unidades oración, enunciado, intervención o turno en su calidad de puras estructuras superficiales. Es decir que no hemos tomado en consideración, ni las estructuras de dependencia jerárquica que a veces se dan entre diferentes elementos de las periferias, ni tampoco con respecto a cuál unidad -oración, enunciado, intervención o turno- un elemento periférico (en adelante: EP) se encuentra a su izquierda o derecha. En la superficie hemos destacado la oración como unidad de base y los EP que preceden, o siguen, al núcleo como simples secuencias conformadas (en nuestros materiales) por uno hasta -en casos extremos de la $\mathrm{Pl}-$ ocho eslabones. En Figura 2 se presentan dos ejemplos, provenientes de nuestros materiales, de cómo hemos procedido en la subdivisión de lo que calificamos de la "oración extendida": 


\begin{tabular}{|c|c|c|}
\hline \multicolumn{3}{|l|}{ Oración extendida } \\
\hline Periferia izquierda & Oración núcleo & Periferia derecha \\
\hline $\begin{array}{l}\text { Ej.1 } \\
\text { (a) diferencias culturales } \uparrow \\
\text { (b) bueno } \rightarrow \\
\text { (c) nosotros Chile } \uparrow\end{array}$ & $\begin{array}{l}\text { tiene mucho mejor nivel } \\
\text { educacional que Brasil } \downarrow\end{array}$ & $\begin{array}{l}\text { (a) a nivel macro } \downarrow \text { (b) } \\
\text { evidentemente } \downarrow\end{array}$ \\
\hline $\begin{array}{l}\text { Ej. } 2 \\
\text { (a) el brasilero } \uparrow \\
\text { (b) en cambio } \rightarrow \\
\text { (c) cuando tiene cincuenta reales } \uparrow\end{array}$ & está feliz de la vida $\downarrow$ & qué sé yo $\downarrow$ \\
\hline
\end{tabular}

Fig. 2. Esquema oracional con núcleo y periferias sintácticas.

Típicamente (aunque no obligatoriamente) se da un límite prosódico entre PI y la oración núcleo, así como entre esta y la PD; igualmente suele darse límites prosódicos ente los diversos eslabones de las periferias respectivas. En los ejemplos de la Figura 2, estos límites están marcados con flechas $(\uparrow, \downarrow, \rightarrow)$.

Debe reconocerse que en un análisis que tomara en cuenta una división jerárquica de las unidades discursivas, el EP "bueno" del primer ejemplo se situaría a la izquierda de la unidad "turno", mientras que el EP "en cambio" en el segundo ejemplo tendría su sitio a la izquierda de la unidad "enunciado". No obstante, para nuestro propósito, que es comparar el peso de las periferias producidas por diversos grupos de hablantes, ha resultados más operacional considerar la PI y la PD como secuencias de EP, o "cadenas de eslabones".

\section{LOS MATERIALES}

Nuestro corpus consta de entrevistas semi-estructuradas representando la actividad (género discursivo) de la autopresentación. El rol del entrevistador en esta actividad es introducir nuevos (sub-)temas y, de manera general, apoyar al entrevistado a producir discurso. La actividad abarca discurso de tipo a la vez monológico (los turnos del entrevistado son prolongados y contiene a menudo elaboradas secuencias narrativas) y diálogico (hay alternancia espontánea entre entrevistado y entrevistador).

Los materiales se reparten como sigue ${ }^{1}$ :

a) 10 entrevistas a hablantes no nativos de español que tienen el sueco como L1,

- realizadas en español

\footnotetext{
${ }^{1}$ Las entrevistas a hablantes de español L1 y L2 provienen de nuestra base de datos original (materiales "Multi-tareas"), mientras las entrevistas a hablantes L1 sueco han sido tomado prestadas de una base de datos del lenguaje oral establecida en Suecia (corpus Swedia 2000, www.umu.se/ forskning/projekt/ swedia-2000).
} 
- con participantes radicados en Chile desde hace 5 años como mínimo (intervalo de 5-19 años de residencia, promedio de 10,4 años)

- su educación: estudios superiores al menos iniciados

- su nivel socioeconómico: entre medio y medio-alto

- distribución etaria: 25-59 años, promedio 39,1 años

- reparto de sexos: $6 \mathrm{~F}, 4 \mathrm{M}$

- Total de palabras: 24 443,

b) 10 entrevistas a hablantes nativos de español chileno,

- realizadas en español

- con variables sociales análogas a las de los entrevistados no nativos

- distribución etaria: 21-71 años (promedio 37,8 años)

- reparto de sexos: $4 \mathrm{~F}, 6 \mathrm{M}$

- Total de palabras: 20253.

c) 5 entrevistas a hablantes nativos de sueco,

- realizadas en sueco

- su educación: enseñanza media o estudios superiores al menos iniciados

- su nivel socioeconómico: medio

- distribución etaria: 21-32 años, promedio 25,8 años

- reparto de sexos: $3 \mathrm{~F}, 2 \mathrm{M}$

- Total de palabras: 14167.

\section{UNIDADES DE LA PERIFERIA IZQUIERDA}

El organigrama de la Figura 3 representa la clasificación de los tipos de EP detectados en la PI:

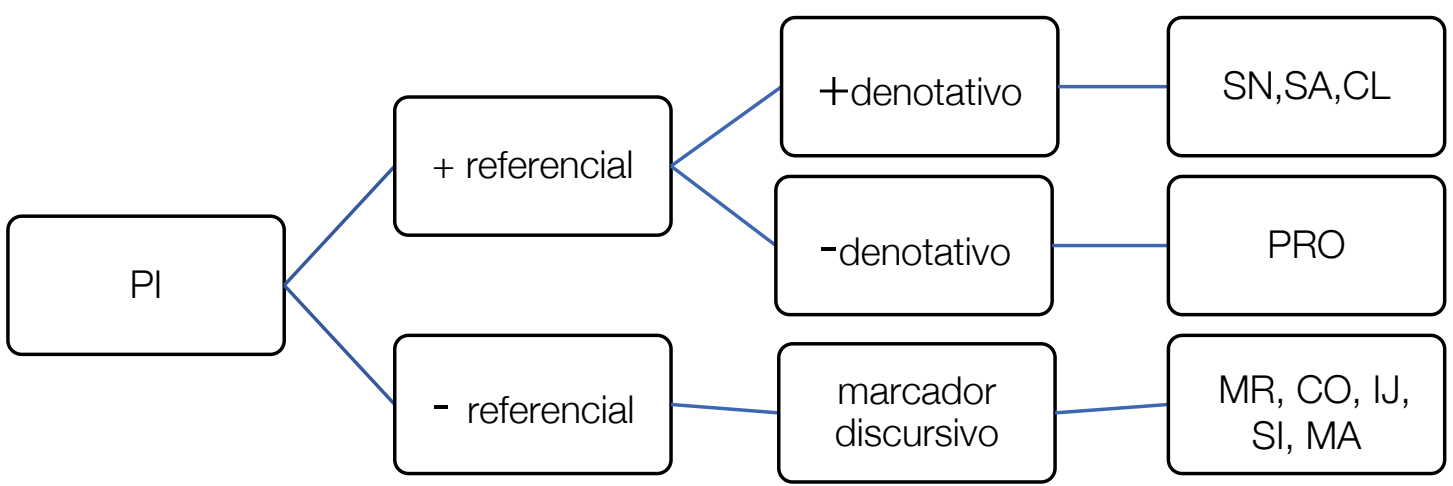

Fig. 3. Tipos de elementos de la periferia izquierda. 
Las periferias sintácticas en el habla coloquial de español L1 y L2 y de sueco L1: un estudio tipológico y adquisicional | L. Fant

La primera subdivisión se hace entre elementos referenciales (conceptuales) y no referenciales (procedimentales). Los EP de la segunda rama los consideramos todos como marcadores del discurso.

En la primera rama, hacemos una división entre EP denotativos --sintagmas nominales (SN), sintagmas adverbiales (SA) y cláusulas, CL)-- y no denotativos, concretamente, los pronombres personales y demostrativos (PRO). Ejemplos de estas cuatro categorías de EP son ${ }^{2}$ :

- SN: en la PI, su función discursiva es la de tópico en el sentido de "cosa comentada", independientemente de su función sintáctica (sujeto, objeto, etc.) o informacional (foco, contraste, tópico independiente, etc.). Van incluidos los sintagmas infinitivos, cláusulas sustantivas y relativas independientes. Ejemplos:

(1) A mí los ingleses [me caen el descueve]. (Sujeto contrastivo.)

(2) Diferencias culturales bueno nosotros Chile [tiene mucho mejor nivel educacional que Brasil] (=Ej. 1; tópico independiente.)

(3) Yo creo que lo que más nos chocó a los dos [es la imposibilidad de decir que no]. (Relativa independiente.)

- SA: estos sintagmas expresan circunstancias temporales, causales, concesivas etc. que son relevantes o necesarias para el entendimiento del núcleo oracional. Ejemplo:

(4) Cada cuatro años [voy a Europa].

- CL: la categoría abarca cláusulas adverbiales así como oraciones de relativo no restrictivas y construcciones con gerundio o participio. En sus funciones semántico-pragmáticas coincide con la categoría de los SA. Ejemplo:

(5) Si es muy fea tampoco [la van a contratar].

- PRO, ejemplo:

(6) A mí los ingleses [me caen el descueve].

En el grupo de EP no referenciales ("marcadores discursivos") distinguimos cinco clases, vgr. conector primario (CO), marcador adverbial otro que conector primario (MA), marcador de respuesta (MR), interjección (IJ) y sintagma iniciador de cláusula (SI).

- CO: su función es conectar el núcleo oracional con el discurso previo. A no ser que le preceda un MR o IJ, ocupa la posición inicial absoluta de la oración. La clase incluye tanto conjunciones iniciadoras de cláusulas adverbiales ("cuando",

\footnotetext{
${ }^{2}$ En adelante, en los ejemplos, el EP ilustrado va en cursiva y el núcleo oracional está puesto entre corchetes.

${ }^{3}$ Todos los ejemplos presentados son auténticos y sacados de nuestro corpus.
} 
Las periferias sintácticas en el habla coloquial de español L1 y L2 y de sueco L1: un estudio tipológico y adquisicional | L. Fant

"ya que", etc.) como conectores adverbiales ("entonces", "por lo tanto", etc.). Ejemplos:

(7) Como yo [conocía el país un poquito]...

(8) Entonces, por lo tanto, el nivel sociocultural [es muy importante].

- MA: es clase híbrida que incluye, entre otros, marcadores epistémicos ("a lo mejor"), evidenciales ("obviamente") y actitudinales/valorativos ("lamentablemente"). Ejemplo:

(9) Obviamente como yo conocía el país un poquito [resultó más fácil para nosotros vivir allá].

Los marcadores de función conectiva que no aparecen en primera posición los consideramos como no primarios; también han sido incluidos en la clase MA:

(10) Entonces, por lo tanto ${ }_{2}$ el nivel sociocultural [es muy importante].

- MR: esta clase conecta con el discurso previo en el plano interaccional, al constituir una reacción positiva ("sí"), negativa ("no") o ambigua ("bueno") a un enunciado anterior producido por el interlocutor (o por el hablante mismo). Ejemplo:

(11) Bueno ahí [tienes otra cosa que me llamó la atención].

- IJ: las interjecciones son categoría de marcador actitudinal considerada como clase morfológica particular. Conecta con el turno/intervención anterior al constituir una reacción al mismo y al determinar la interpretación del discurso que sigue:

(12) Uf ee como te digo [no he alcanzado a conocer a tantos ingleses].

- SI (sintagma iniciador de cláusula, o "botador de oración", sentence launcher): aunque en el plano formal los SI son oraciones principales con complemento oracional, desde la perspectiva pragmática pueden considerarse como elementos en plan de igualdad con los MA de significado epistémico, evidencial o actitudinal. Ejemplo:

(13) Yo creo que lo que más nos chocó a los dos [es la imposibilidad de decir que no].

El ejemplo anterior puede ser comparado con el siguiente:

(14) Seguramente lo que más nos chocó a los dos [es la imposibilidad de decir que no].

\section{UNIDADES DE LA PERIFERIA DERECHA}

El organigrama de la Figura 4 representa la clasificación de los tipos de EP que están situados en la periferia derecha: 


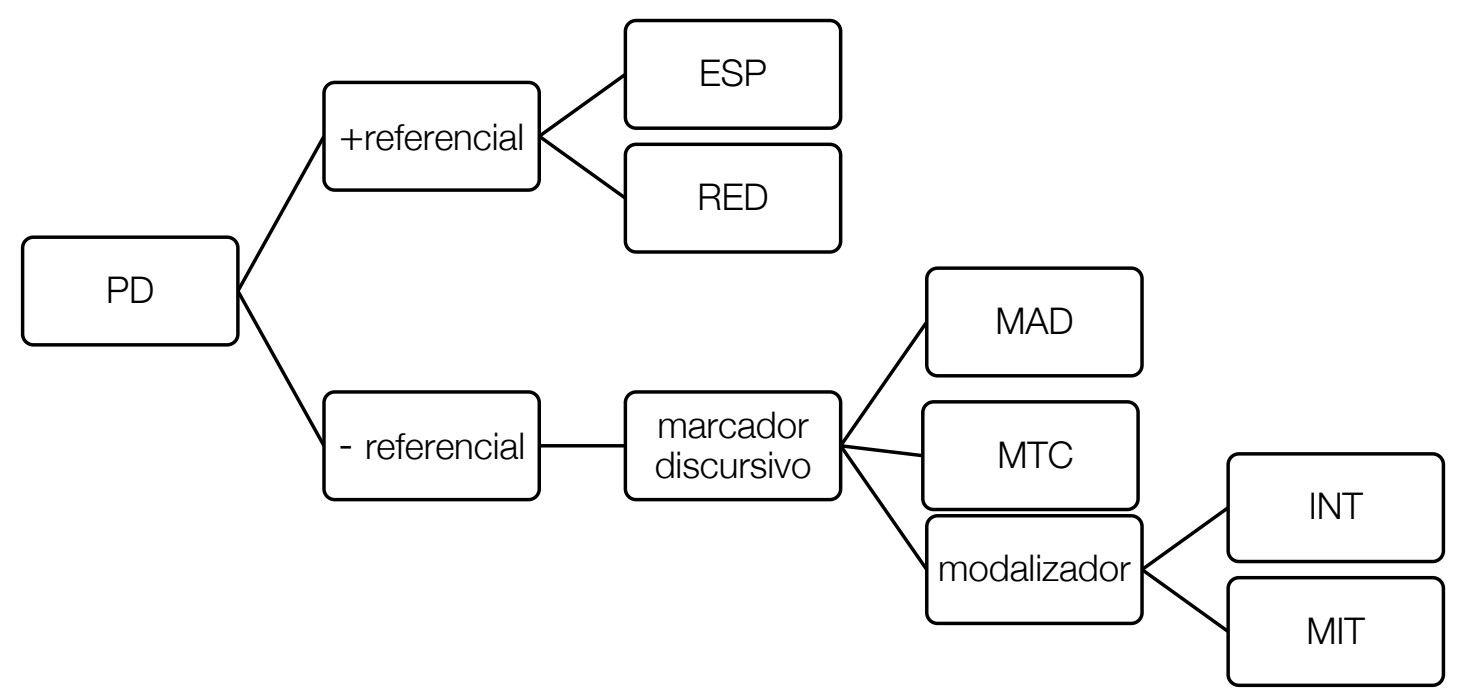

Fig. 4. Tipos de elementos de la periferia derecha.

Al igual que para la periferia izquierda, nuestra primera subdivisión de los EP de la periferia derecha se hace entre elementos referenciales (conceptuales) y no referenciales (procedimentales). Entre los primeros distinguimos los especificadores (ESP) de los reduplicadores (RED).

- ESP: los especificadores son sintagmas nominales, adverbiales o cláusulas (enteros o parciales) con función reparadora, suministrando elementos que se han de entender como ausentes en el tramo de discurso que le precede. Ejemplo:

(15) [Y ahora trabajo en la Comisión Europea $\downarrow]$ en la embajada en Santiago.

- RED: un elemento reduplicador reitera, por diferentes motivos y de manera más o menos literal, elementos ya presentes en el enunciado. Ejemplos:

(16) Después [conocí a mi actual esposa ee $\rightarrow$ ] mi señora.

(17) ....además de que [dejé buenos amigos allá $\downarrow$ ] muy buenos amigos.

Entre las expresiones procedimentales/no referenciales destacamos seis clases: marcadores mitigadores (MIT), intensificadores (INT), aditivos (MAD) y marcadores de terreno compartido (MTC).

Los mitigadores e intensificadores son marcadores modalizadores que sirven el propósito de atenuar y reforzar, respectivamente, la fuerza pragmática del enunciado. Son funciones subjetivas más bien que intersubjetivas: 
Las periferias sintácticas en el habla coloquial de español L1 y L2 y de sueco L1: un estudio tipológico y adquisicional | L. Fant

- MIT: entre los mitigadores hay varios subtipos, como p.ej. los resguardos y barreras ("por así decirlo") o las señales de incompletitud ("y todo y tal”). Ejemplo:

(18) [Y son los encargados del comercio exterior de Chile] digamos.

- INT: los intensificadores producen el efecto contrario, bien sea por recalcar la importancia de (parte de)l significado del enunciado, o por señalar, por ejemplo, que lo que dice el emisor es para tomado en serio. Ejemplo:

(19) [No es un lugar que elegiría para vivir para retirarme] de todas maneras.

Un tercer grupo de elementos procedimentales /no referenciales de la EP son los marcadores aditivos (MAD):

- MAD: un subtipo de esta categoría es el que, al igual que los conectores de la PI, establece enlace con el discurso anterior. Se trata de expresiones como "también", "además" o "por ejemplo":

(20) Creo que [hay un choque muy grande por el tema de la formalidad] por ejemplo.

Otro subtipo de los MAD consta de conectores que quedan "pendientes" al final de un enunciado. Ejemplo:

(21) [...y que teníamos muchos amigos comunes] así que $\rightarrow \ldots$

(22) [...en otros momentos estoy mirando los cerros] ahi $\rightarrow$ ee irme a los cerros estar dos o tres días.

El último tipo de elementos procedimentales/no referenciales que queremos destacar son los marcadores de terreno compartido (MTC). Es categoría destinada a establecer intersubjetividad, al igual que los marcadores de respuesta (MR) de la PI, la diferencia siendo que los MR son elementos reactivos dirigidos al tramo de discurso anterior, mientras los MTC son elementos iniciativos destinados para establecer terreno interaccional compartido. Existen dos tipos: "afirmar terreno compartido" (por defecto señalado mediante un tono final descendente) y "solicitar terreno compartido" (por defecto señalado mediante un tono final ascendente).

- MTC de afirmación, ejemplo:

(23) Y como tiene alcohol [se van evaporando] po $\downarrow$

(24) [Pero es una empresa brasilera] ya $\downarrow$

- MTC de solicitud, ejemplo:

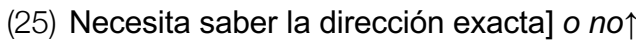


Las periferias sintácticas en el habla coloquial de español L1 y L2 y de sueco L1: un estudio tipológico y adquisicional | L. Fant

\section{Resultados}

\subsection{La periferia izquierda}

A. Con respecto a los usos de la PI, el español L1 difiere significativamente del sueco L1. La mayoría de las divergencias son estadísticamente significativas:

- Los hablantes de sueco L1 producen, en total, un menor número de EP: 8,5 instancias por 100 palabras frente a 10,95 instancias por 100 palabras en el grupo español L1 $\left(p==0,0007^{* * *}\right)$.

- Los hablantes de sueco L1 producen menos sintagmas adverbiales (SA): 0,42 instancias por 100 palabras, frente a 0,95 instancias por 100 palabras en el grupo español L1 $\left(p=0,0003^{* * *}\right)$.

- Los hablantes de sueco L1 producen menos cláusulas (CL): 0,30 instancias por 100 palabras, frente a 0,62 instancias por 100 palabras en el grupo español L1 $\left(p=0,007^{\star *}\right)$.

- Los hablantes de sueco L1 producen menos sintagmas iniciadores de cláusula (SI): 0,12 instancias por 100 palabras frente a 0,49 instancias por 100 palabras en el grupo español L1 $\left(p=0,006^{* *}\right)$

Una posible explicación de estas divergencias reside en la regla V2 de la lengua sueca, así como de otras lenguas germánicas ("siempre colocar el verbo en segunda posición"), regla que reduce la posibilidad de colocar elementos referenciales en la PI. Además, la posición medial fija asignada a varios tipos de marcadores discursivos (rasgo común también para varias lenguas germánicas) impide la colocación de estos en la PI.

En cambio, si comparamos la actuación de los hablantes nativos de español con aquella de los no nativos avanzados, las similitudes saltan al ojo. El hecho de que las cifras de uno y otro grupo coincidan en grado sorprendente supone un alto grado de alineamiento por parte de los no nativos.

No obstante, hay dos diferencias interesantes:

- Los hablantes de español L2 tienden a "sobreusar" pronombres en comparación con los hablantes nativos: 2,48 instancias por 100 palabras frente a 2,05 instancias por 100 palabras en el grupo de hablantes nativos ESP-L1 ( $p=0,07$-resultado no enteramente significativo).

- En cambio, los hablantes de español L2 "subusan" los sintagmas nominales $(1,25$ instancias por 100 palabras frente a 1,62 instancias por 100 palabras en los ESP-L $1 ; p=0,004^{*}$ ).

Lo observado puede ser explicado como un rasgo característico del uso de la L2, a saber, que sería más "fácil" -o representaría un coste menor- recurrir a pronombres que usar expresiones denotativas, lo cual supondría una búsqueda léxica que se diera de forma más automática en el hablante nativo que en el no nativo. 
Las periferias sintácticas en el habla coloquial de español L1 y L2 y de sueco L1: un estudio tipológico y adquisicional | L. Fant

B. También deben compararse las producciones en español L2 con las del sueco L1, con vistas a poder rastrear instancias de transferencia de la lengua nativa a la no nativa. El análisis resulta bastante decepcionante para quienes defienden la postura de que es imposible para un no nativo llegar a niveles nativos: aquí se dan las mismas discrepancias que entre sueco L1 y español L1. En otras palabras: no es posible detectar instancias claras de transferencia de la L1 a la L2.

\subsection{La periferia derecha.}

A. Al compararse los resultados del grupo de español L1 y L2 con los del grupo de sueco $L 1$ el análisis arroja resultados en parte coincidentes con los relativos a la PI.

Así, los hablantes de sueco L1 producen en total un número considerablemente más alto de EP, no solo comparado con los hablantes de español $L 1$, sino también en comparación con los hablantes de español L2: llegan a 4,17 instancias por 100 palabras, frente a 1,34 instancias por 100 palabras en el grupo de español $L 1\left(p=0,007^{* *}\right)$ y 1,44 instancias por 100 palabras en el grupo de español L2 $\left(p=0,008^{* *}\right)$. Esto, junto con el correspondiente resultado del análisis de la PI, nos corrobora en la convicción de que existe una diferencia tipológica entre lenguas que tienden hacia la periferización a la derecha (como el sueco) y las que prefieren la periferización a la izquierda (como el español y demás lenguas románicas). Debe subrayarse, sin embargo, que esta divergencia se hace notar más en la PD que en la PI.

Lo dicho arriba describe la tendencia global. No obstante, al hacer un análisis del uso de las categorías específicas de EP específicos, se notan diferencias interesantes. Así, los hablantes de sueco L1 producen un mayor número de EP que los de español L1 y L2 en tres de las categorías no referenciales ${ }^{4}$ :

- $\quad$ MTC: 1,91 instancias por 100 palabras frente a 0,49 instancias por 100 palabras en el grupo de español L1 $\left(p=0,012^{*}\right)$ y 0,40 instancias por 100 palabras en el grupo de español L2 $\left(p=0,0099^{* *}\right)$

- MIT: 1,28 instancias por 100 palabras frente a 0,35 instancias por 100 palabras en el grupo español L1 $\left(P=0,005^{* *}\right)$ y 0,39 instancias por 100 palabras en el grupo de español L2 $\left(p=0,002^{* * *}\right)$

- INT: 0,52 instancias por 100 palabras frente a 0,10 instancias por 100 palabras en el grupo español L1 $\left(p=0,037^{*}\right)$ y 0,11 instancias por 100 palabras en el grupo de español L2 $\left(p=0,032^{*}\right)$.

Hasta qué punto estas divergencias radican en preferencias socio-culturales o en características estructurales de una y otra lengua, queda una cuestión hasta ahora abierta. También hay que apreciar hasta qué punto las funciones pragmáticas en

\footnotetext{
${ }^{4}$ En lo que se refiere a la cuarta categoría no referencial de la PD, a saber, los marcadores aditivos (MAD), la tendencia es la inversa: los hablantes de sueco L1 producen un menor número de estos elementos que los dos otros grupos: 0,27 instancias por 100 palabras frente a 0,7 instancias por 100 palabras en el grupo español L1 y 0,8 instancias por 100 palabras en el grupo de español L2. Estas cifras no son enteramente significativas estadísticamente, ni tampoco se prestan a una interpretación inequívoca.
} 
Las periferias sintácticas en el habla coloquial de español L1 y L2 y de sueco L1: un estudio tipológico y adquisicional | L. Fant

cuestión se realizan fuera de la periferia derecha, a saber, en el núcleo oracional o en la periferia izquierda.

B. Si comparamos las cifras del grupo de español $L 1$ con las del grupo $L 2$, en ningún caso se da una discrepancia notable. Por otra parte, al compararse los resultados del grupo de español L2 con el del grupo sueco L1, podemos constatar que se dan importantes discrepancias entre suecos que hablan su lengua nativa y los que son usuarios proficientes de español como L2. Debemos por lo tanto sacar la misma conclusión que para la PI: es difícil comprobar efectos de transferencia desde la L1 a la L2, cuando se trata de usuarios tan avanzados como los de nuestro corpus.

Una tendencia, sin embargo, se hace visible, no solo al tratarse de las categorías de la PD sino también de las de la PI: mientras los valores recogidos en el grupo español L1 por lo general se ven muy cercanos a los del grupo español L2, siempre y cuando difieren, aunque la divergencia sea mínima, el valor del grupo español L2 se aproxima, por muy poco que sea, al valor correspondiente del grupo sueco L1. Parece por lo tanto producirse en los hablantes no nativos un efecto de atracción -ligero o subliminal- de parte de la lengua nativa.

\section{A MODO DE CONCLUSIÓN}

Como respuesta a la primera pregunta de investigación, relativa a la tipología, queda claro que existen importantes diferencias estructurales relativas a las periferias sintácticas entre el español y el sueco. Así los hablantes nativos de sueco periferizan hacia la derecha considerablemente más que los hablantes nativos de español. Estos, por el contrario, periferizan más hacia la izquierda que los suecos, aunque la desproporción no sea tan grande como en el caso de la periferia derecha. En total, los suecos en su lengua nativa periferizan en mayor grado que los hablantes nativos de español.

En lo que se refiere a la segunda pregunta de investigación, relativa al proceso de adquisición de segundas lenguas, hemos podido ver, por un lado, que el comportamiento de los hablantes no nativos de español es muy parecido y a veces idéntico al del grupo de hablantes nativos. Por otro lado, se pueden constatar importantes divergencias entre los resultados del grupo sueco $L 1$ y los del grupo español L2, lo cual nos hace concluir que el efecto de transferencia de la L1 es muy escaso en estos usuarios muy avanzados de español como segunda lengua.

Las dos diferencias comprobadas entre los grupos nativo y no nativo de español, a saber, la tendencia no nativa hacia un sobreuso en la PI de pronombres, por un lado, y el subuso de sintagmas nominales, por el otro, podrían ser explicadas, no como efectos de transferencia de la L1 sino como reflejo general de una inseguridad producida al hablar una lengua no nativa (ver p.ej. Andersen and Shirai, 1994: 145-46), la cual por lo visto puede darse aun en hablantes muy avanzados de una L2.

\section{REFERENCIAS}


Andersen, Roger W. y Yasuhiro Shirai (1994): «Discourse motivations for some cognitive acquisition principles», Studies in Second Language Acquisition 16, 133-156.

Bañeras Carrió, María (2016): La periferia izquierda de la oración. Basis de una propuesta configuracional, tesis del grado, Universitat Autònoma de Barcelona.

Bartning, Inge, Fanny Forsberg y Victorine Hancock (2012): «On the role of contextual factors for morpho-syntactic stabilization in high level L2 French», Studies in Second Language Acquisition, 34 (2), 243-267.

Beeching, Kate y Ulrich Detges, eds. (2014): Functions at the left and right periphery: Crosslinguistic investigations of language use and language change, Leiden: Brill.

Beeching, Kate, Liesbeth Degand, Ulrich Detges, Elizabeth Closs Traugott y Richard Waltereit (2009): Summary of the Workshop on Meaning in Diachrony at the Conference on Meaning in Interaction, University of the West of England (Bristol).

Briz Gómez, Antonio y Grupo Val.Es.Co (2003): «Un sistema de unidades para el estudio del lenguaje coloquial», Oralia 6, 7-61.

Cinque, Guglielmo y Luigi Rizzi (2008), «The Cartography of Syntactic Structures», Studies in Linguistics / CISCL Working Papers Vol. 2, 42-58.

Demonte ,Violeta y Olga Fernández Soriano (2007), «La periferia izquierda oracional y los complementantes del español», en Cuartero Otal, Juan y Martina Emsel (eds.), Vernetzungen: Bedeutung in Wort, Satz Und Text, Frankfurt: Peter Lang, 1-13.

Detges, Ulrich y Richard Waltereit (2014): «Moi je ne sais pas vs. Je ne sais pas moi: French disjoint pronouns in the left vs. right periphery», en Beeching, Kate y Ulrich Detges, eds., Discourse functions at the left and the right periphery. Crosslinguistic investigations of language use and language change, Leiden: Brill, 24-46.

Estellés Arguedas, María y Salvador Pons Bordería (2014): «Absolute initial position», en Pons Bordería, Salvador, ed., Discourse Segmentation in Romance Languages, Amsterdam/Philadelphia: John Benjamins, 121-155.

Hancock, Victorine (2007): «Quelques éléments modaux dissociés dans le paragraphe oral dans des interviews en français $L 2$ et $L 1 »$, Journal of French Language Studies 17, 2147.

Hendriks, Henrietta y Marzena Watorek, (2008): «L'organisation de l'information en topique dans les discours descriptifs en L1 et L2», AILE 26, 129-148.

Hyltenstam, Kenneth, Inge Bartning y Lars Fant eds. (2018): High level proficiency in second languages and multilingual contexts, Cambridge: Cambridge University Press.

Kotschi, Thomas (1996): «Procedimientos de producción y estructura informacional en el lenguaje hablado», en Kotschi, Thomas, Wolf Oesterreicher y Klaus Zimmermann, eds., El español hablado y la cultura oral en España e Hispanoamérica, Frankfurt am Main: Vervuert/ Madrid: Iberoamericana, 185-206.

Lambrecht, Knud (1994): Information structure and sentence form. Topic, focus and the mental representations of discourse referents, Cambridge: CUP.

Pons Bordería, Salvador (en prensa): Paths of grammaticalization: beyond the LP/RP debate, Universitat de València, Grupo Val.Es.Co.

Rizzi, Luigi (1997): «The Fine Structure of the Left Periphery», en Haegeman, Liliane (ed.), Elements of Grammar, Dordrecht: Kluver, 281-337.

Salameh Jiménez, Shima, Maria Estellés Arguedas y Salvador Pons Bordería (2018): «Beyond the notion of periphery: An account of polyfunctional discourse markers within the Val.Es.Co model of discourse segmentation», en Beeching, Kate, Chiara Ghezzi y Piera Molinelli (eds.), Positioning the self and others: Linguistic traces, Amsterdam/Philadelphia: John Benjamins, 105-125.

Sedano, Mercedes (2012): «Dislocación a la izquierda y a la derecha: semejanzas y diferencias», Homenaxes, 58, Departamento de Lingua Española, Servizo de Publicacións e Intercambio Científico da Universidade de Santiago de Compostela, 791-805.

Sorace, Antonella (2003): «Near-nativeness», en Doughty, Catherine y Michael Long, eds., Handbook of Second Language acquisition, Oxford: Blackwell, 130-151.

Stutterheim, Christiane von (2003): «Linguistic structure and information organisation. The case of very advanced learners», EUROSLA Yearbook 3, 183-206.

Traugott, Elizabeth Closs (2012): «Intersubjectification and clause periphery», English Text Construction, 5:1.

Traugott, Elizabeth Closs (2014): «On the Function of the epistemic adverbs Surely and No doubt at the left and right peripheries of the clause», en Beeching, Kate y Ulrich Detges, eds., Discourse functions at the left and right periphery, Leiden/Boston: Brill, 72-91. 
Las periferias sintácticas en el habla coloquial de español L1 y L2 y de sueco L1: un estudio tipológico y adquisicional | L. Fant 